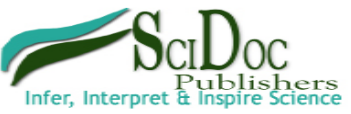

International Journal of Bioorganic Chemistry \& Molecular Biology (IJBCMB)

ISSN 2332-2756

\title{
HDAC Inhibitors and HAT Activators - Different Activity, Same Outcome
}

Editorial

Hari Kishore A

The Cecil H. and Ida Green Center for Reproductive Biology, Department of Obstetrics and Gynecology, University of Texas Southwestern Medical Center, Dallas, Texas, USA.

\section{*Corresponding Author:}

Annavarapu Hari Kishore,

The Cecil H. and Ida Green Center for Reproductive Biology, Department of Obstetrics and Gynecology, University of Texas Southwestern Medical Center, Dallas, Texas, USA.

Tel: 001-2146486809

E-mail: hari.annavarapu@utsouthwestern.edu

Received: June 13, 2014

Published: June 25, 2014

Citation: Hari Kishore A (2014) HDAC Inhibitors and HAT Activators - Different Activity, Same Outcome. Int J Bioorg Chem Mol Biol. 2(3e), 1-2. doi: http://dx.doi.org/10.19070/2332-2756-140006e

Copyright: Hari Kishore $\mathbf{A}^{\mathcal{O}}$ 2014. This is an open-access article distributed under the terms of the Creative Commons Attribution License, which permits unrestricted use, distribution and reproduction in any medium, provided the original author and source are credited.

Histone acetyltransferases (HATs) are a group of enzymes that acetylate lysine residues in histones, which are bound to DNA in all eukaryotes to form a complex and well organized structure called chromatin. HATs play an important role in regulating the chromatin organization and accessibility of the DNA to the transcriptional machinery by altering the acetylation status of histones in particular gene loci by chromatin remodeling. Histone acetylation marks active gene transcription while lack of acetylation marks gene repression. The -acetyl moieties are removed from the histones by Histone deacetylases (HDACs). Thus HATs and HDACs play a dynamic role in switching a gene ON and OFF. Dysfunction and deregulation of these enzymes leads to aberrant expression of genes thereby affecting the cellular phenotype, resulting in diseases like cancer and neurodegenerative disorders $[1,2]$. Several proteins including transcription factors also undergo dynamic acetylation and deacetylation processesthat affects protein-protein interactions, protein-DNA interactions and cellular localization thereby changing the physiology of the cell. Thus in several disease models HATs and HDACs are attractive targets for manipulating the gene expression by altering their activity by small molecule modulators.

In cancerous cells histone acetylation pattern in the chromatin is deregulated due to either i) improper targeting of HATs and HDACs to a particular gene loci, ii) overexpression of HDACs or iii) inactivation of HAT activity due to mutations thereby causing aberrant gene expression. Restoring the expression of genes aberrantly suppressed, required for normal cell physiology is the basis for development of HDAC inhibitors as cancer therapeutics. Though acetylation/deacetylation determines the accessibility of DNA to transcription machinery in chromatin, HDAC inhibitors are known to affect only around $10 \%$ of genes which importantly include genes that regulate cell cycle and apoptosis [1]. This ability makes them non-toxic to normal cells and hence attractive agents in chemotherapy. Several HDAC inhibitors like Romidepsin, SAHA, LBH589, Valproic acid, TSA etc., are in various stages of clinical trials and hold promise as future drugs to treat cancer.

Recently Histone acetylation in brain cells has been linked to neuro-protection and memory functions. Several neurological disorders like Huntington's disease (HD), Parkinson's disease (PD), Alzheimer's disease (AD) and Amyotrophic lateral sclerosis (ALS) are all associated with histone hypoacetylation. HD is characterized by progressive neurodegeneration (loss of neurons) that is linked to the product (PolyQ htt) of CAG repeat expansion in Huntingtin gene sequestering and inhibiting HATs- p300 and CBP. Several cellular and animal HD-models have shown to be associated with decreased Histone $\mathrm{H} 3$ acetylation level (in particular H3K9 and H3K14) [3]. In PD $\alpha$-synuclein, a nuclear protein promotes neurotoxicity by binding and masking histones thereby blocking acetylation [4]. AD is associated with abnormal acetylation of Tau protein and over expression of amyloid $\beta$ precursor protein (APP) that leads to decreased CBP levels and thereby reduced histone $\mathrm{H} 3$ and $\mathrm{H} 4$ acetylation [5]. Histone deacetlylases are shown to be aberrantly upregulated in neurons of brain from ALS patients [6]. Albeit the differences in pathophysiology, aberrant acetylation/deacetylation seems to be the hallmark of neurological disorders. Thus HDAC inhibitors were considered as therapeutics in order to restore the acetylation levels and thereby gene expression in neuropathic conditions. Recent studies have implicated histone epigenetic modifications, in particular histone acetylation, in learning, memory retrieval and synaptic plasticity. In animal models Histone acetylation levels were shown to increase in hippocampus immediately after training. In animal models systemic administration of HDAC inhibitor - Sodium Butyrate was shown to enhance memory while intrahippocampal injection of TSA immediately after training enhanced long term memory further confirming the positive role of histone acetylation in gene regulation required for learning and memory in brain.

Despite several studies and clinical trials with HDAC inhibitors as therapeutic interventions for various cancers and neurological disorders, scientists are skeptical about their use because of their non-specific nature and detrimental effects of long term use. Also there are as many as eighteen HDACs identified to date with functional redundancy and often associated as a complex rather than functioning individually which makes specific targeting impossible. Recently a new class of molecules - "HAT activators" has been discovered with ability to induce hyperacetylation by activating HATs. 
An amide derivative of HAT inhibitor Anacardic acid - N-(4chloro-3-trifluoromethyl-phenyl)-2-ethoxy-6-pentadecyl-benzamide (CTPB) was shown to specifically activate p300 but not p300/CBP-associated factor (PCAF) in vitro. However, the use of CTPB to activate HATs in vivo was not possible because of its cell permeability, which was overcome by nanoparticle delivery [7]. Further an analog of CTPB, N-(4-chloro-3-trifluoromethylphenyl)-2-ethoxy-benzamide (CTB) that lacks the pentadecyl -side chain was shown to alter the structure of p300 thereby enhancing its enzymatic activity [8]. CTB was also shown to increase acetylation of p53 in MCF7 breast cancer cells. Nemorosone a polyisoprenylated benzophenone from natural sources was shown to selectively bind to and enhance the acetyltransferase activity of p300/CBP in vitro as well as in cell culture models [9]. Pentadecylidenemalonate a simple derivative of Anacardic acid was shown to enhance PCAF activity while inhibiting p300/CBP in vitro. [10] Remarkably cell permeability, HAT enhancing ability and anti-proliferative activity of CTB, Nemorosone and Pentadecylidenemalonate make them attractive leads for development of anticancer drugs. Recently, TTK21 a p300/CBP activator, conjugated to glucose based carbon nanosphere when administered intra-peritoneal enhanced histone acetylation in hippocampus the main learning and memory center and frontal cortex of brain. Incidentally hippocampus is the first part of brain to undergo neurodegeneration in AD. TTK21 enhanced differentiation and maturation of hippocampal neurons thereby promoting long term memory [11].

HAT activators and HDAC inhibitors have the potential to become future therapeutics playing crucial role in restoring histone acetylation levels in disease conditions associated with hypoacetylation. Also, it would be interesting to study the synergistic effects of HAT activators and HDAC inhibitors in order to achieve minimum toxicity and optimal effect which requires identification and characterization of bigger library of small molecules to choose from.

\section{References}

[1]. Johnstone RW. (2002) Histone-deacetylase inhibitors: novel drugs for the treatment of cancer. Nat Rev Drug Discov 1:287-99.

[2]. Selvi BR, Cassel JC, Kundu TK, Boutillier AL. (2010) Tuning acetylation levels with HAT activators: therapeutic strategy in neurodegenerative diseases. BiochimBiophysActa. 1799:840-53.

[3]. McFarland KN, Das S, Sun TT, Leyfer D, Xia E, et al. (2012) Genome-wide histone acetylation is altered in a transgenic mouse model of Huntington's disease.PLoS One 7:e41423.

[4]. Kontopoulos E, Parvin JD, Feany MB. (2006) Alpha-synuclein acts in the nucleus to inhibit histone acetylation and promote neurotoxicity. Hum Mol Genet 15:3012-23.

[5]. Francis YI, Fà M, Ashraf H, Zhang H, Staniszewski A,et al (2009) Dysregulation of histone acetylation in the APP/PS1 mouse model of Alzheimer's disease. J Alzheimers Dis. 18:131-9.

[6]. Janssen C, Schmalbach S, Boeselt S, Sarlette A, Dengler R, et al. (2010) Differential histone deacetylase mRNA expression patterns in amyotrophic lateral sclerosis. J NeuropatholExp Neurol 69:573-81.

[7]. Balasubramanyam K, Swaminathan V, Ranganathan A, Kundu TK. (2003) Small molecule modulators of histone acetyltransferase p300. J Biol Chem 278:19134-40

[8]. Mantelingu K, Kishore AH, Balasubramanyam K, Kumar GV, Altaf M, et al. (2007) Activation of p300 histone acetyltransferase by small molecules altering enzyme structure: probed by surface-enhanced Raman spectroscopy.J PhysChem B 111:4527-34.

[9]. Dal Piaz F, Tosco A, Eletto D, Piccinelli AL, Moltedo O, et al. (2010) The identification of a novel natural activator of $\mathrm{p} 300$ histone acetyltranferase provides new insights into the modulation mechanism of this enzyme. Chembiochem 11:818-27.

[10]. Sbardella G, Castellano S, Vicidomini C, Rotili D, Nebbioso A, et al. (2008) Identification of long chain alkylidenemalonates as novel small molecule modulators of histone acetyltransferases. Bioorg Med ChemLett. 18:278892.

[11]. Chatterjee S, Mizar P, Cassel R, Neidl R, Selvi BR, et al. (2013) A novel activator of $\mathrm{CBP} / \mathrm{p} 300$ acetyltransferases promotes neurogenesis and extends memory duration in adult mice. J Neurosci 33:10698-712. 